\title{
MODELO DE PROGRAMAÇÃO LINEAR PARA SELEÇÃO DE PULVERIZADORES AGRÍCOLAS DE BARRAS
}

\author{
FÁBIO H. R. BAIO ${ }^{1}$, ULISSES R. ANTUNIASSI ${ }^{2}$, LUIZ A. BALASTREIRE ${ }^{3}$, \\ JOSÉ V. CAIXETA FILHO
}

\begin{abstract}
RESUMO: A seleção de pulverizadores agrícolas que se adaptem às necessidades da propriedade, é um processo trabalhoso, sendo uma das etapas mais importantes dentro do processo produtivo. $\mathrm{O}$ objetivo do presente trabalho foi o de desenvolver e utilizar um modelo de programação linear para auxiliar na seleção de pulverizadores agrícolas de barras, baseado no menor custo horário do equipamento. Foram utilizadas as informações técnicas referentes a 20 modelos de pulverizadores disponíveis no mercado, sendo quatro autopropelidos, oito de arrasto e oito do tipo montado. A análise de sensibilidade dos componentes dos custos operacionais mostrou que as taxas de reparo e depreciação foram os fatores que mais interferiram na variação do custo horário do conjunto tratorpulverizador. $\mathrm{O}$ modelo matemático desenvolvido facilitou a realização da análise de sensibilidade que foi processada em um tempo muito pequeno.
\end{abstract}

PALAVRAS-CHAVE: programação linear, tratamento fitossanitário, máquinas agrícolas.

\section{LINEAR PROGRAMMING MODEL TO AGRICULTURAL BOOM SPRAYER SELECTION}

SUMMARY: The selection process of agricultural machines is one of the most important stages of the productive process. The purpose of the present work was to develop and to test a linear programming model to aid in the decision of agricultural boom sprayer selection based on the cheapest hour-cost. The technical information about 20 sprayer models available in the market was used, where four were self-propelled, eight were trailed and eight were lift mounted sprayer. The sensibility analysis of the operational cost components showed that the repair and depreciation rates are the factors that most contribute to hour-cost variation of the sprayer-tractor group. The developed mathematical model facilitated the accomplishment of the sensibility analysis, which was processed in a very short time.

KEYWORDS: linear programming, phytosanitary treatment, agricultural machines.

\section{INTRODUÇÃO}

A seleção de uma máquina agrícola, bem como a de um implemento, pode tornar-se uma tarefa árdua, pois há diversas variáveis que devem ser consideradas, e a escolha do equipamento mais adequado para uma propriedade agrícola é uma das etapas mais importantes do processo produtivo. Modelos matemáticos podem ser considerados como ferramentas úteis na seleção de implementos agrícolas.

Para SOFFNER et al. (1993), a maximização da lucratividade do negócio agrícola é o que determina o gerenciamento ótimo da maquinaria agrícola e não o contrário, pois a simples minimização dos custos das máquinas não é condição suficiente para a maximização do lucro do

\footnotetext{
${ }^{1}$ Aluno de Doutorado em Energia na Agricultura, Faculdade de Ciências Agronômicas - UNESP, Departamento de Engenharia Rural, Botucatu - SP, Fone: (0XX14) 6802.7165, e-mail: fbaio@fca.unesp.br

${ }^{2}$ Professor Doutor, Faculdade de Ciências Agronômicas - UNESP, Botucatu - SP.

${ }^{3}$ Professor Titular, Escola Superior de Agricultura "Luiz de Queiroz" - USP, Piracicaba - SP.

${ }^{4}$ Professor Doutor, Escola Superior de Agricultura "Luiz de Queiroz" - USP, Piracicaba - SP

Recebido pelo Conselho Editorial em: 30-4-2002

Aprovado pelo Conselho Editorial em: 18-8-2003
} 
sistema. De acordo com os autores, exigências da cultura, como a pontualidade na execução das operações, podem demandar sistemas mecanizados com custo superior ao mínimo para que a otimização seja atingida.

Segundo WILLIAMS (1993), o termo modelo é normalmente utilizado para caracterizar estruturas que representam feições e objetos ou cenários. Como explana CAIXETA FILHO (2001), um modelo matemático é uma estrutura que procura representar a realidade simplificadamente. Muitas vezes, os modelos matemáticos são elaborados com o objetivo de escolher uma solução ótima. Segundo HILLER \& LIEBERMAN (1988), uma solução ótima é uma solução viável que possui o valor mais favorável para a função-objetivo, dependendo se o objetivo é maximizar ou minimizar a função. A obtenção da solução ótima é dependente das variáveis de decisão, as quais podem estar sujeitas a uma série de restrições ou limitações.

De acordo com HILLER \& LIEBERMAN (1988) e WILLIAMS (1993), a programação linear é uma ferramenta para o planejamento de atividades para a obtenção de um resultado ótimo, respeitando as alternativas viáveis. Em um problema de programação linear, geralmente, há algumas soluções viáveis, quando todas as restrições são satisfeitas; no entanto, há somente uma solução ótima. Há alguns problemas reais em que a solução somente faz sentido se as variáveis de decisão possuírem valores inteiros, como na seleção de máquinas agrícolas. Para HILLER \& LIEBERMAN (1988), um problema de programação linear inteira é aquele que está sujeito a uma restrição adicional, em que as variáveis de decisão devem possuir valores inteiros.

BARBOZA et al. (1997) criaram um modelo computacional para a análise de um sistema mecanizado agrícola, com o objetivo de identificar os fatores críticos que interferem no custo de produção. Verificaram que os custos referentes à mecanização das operações agrícolas podem chegar a $40 \%$ dos custos de produção, demonstrando a importância da escolha do equipamento mais adequado para o processo produtivo. Os autores observaram que o custo para a aquisição dos equipamentos e o número de horas de utilização dos mesmos ao ano foram os fatores do modelo que mais interferiram no custo final. ROTZ (1986) criou um modelo para avaliar a participação dos fatores reparo e manutenção de equipamentos agrícolas no custo operacional dos mesmos. MERCANTE et al. (2001) elaboraram um programa computacional para a seleção e a estimativa do custo operacional de máquinas e implementos agrícolas. LOPES et al. (1995) desenvolveram um programa computacional para selecionar um sistema mecanizado agrícola, baseado no menor custo operacional. FEY et al. (2002) utilizaram um modelo de programação linear com o objetivo de otimizar um sistema agrícola visando à maximização do seu lucro. Os autores consideraram as restrições de terras, rotação de culturas, recursos financeiros e maquinários agrícolas disponíveis. A resolução do modelo possibilitou o aumento do lucro da propriedade em $9,8 \%$.

O objetivo do presente trabalho foi o de desenvolver e utilizar um modelo de programação linear para auxiliar na seleção de pulverizadores agrícolas de barras, baseado no menor custo horário do equipamento.

\section{MATERIAL E MÉTODOS}

Foi desenvolvido um modelo de programação linear binário para o auxílio no processo de seleção de pulverizadores de barras. Para a aplicação do modelo, foi considerada uma propriedade agrícola hipotética situada na região de Ribeirão Preto - SP, que cultiva 1.000 ha de milho (Zea mays) e soja (Glycine max). Foi considerado que a soja necessita de uma pulverização de herbicida na época da semeadura e de duas pulverizações de inseticidas durante o seu desenvolvimento. Também considerou-se que a cultura do milho necessita de uma pulverização de herbicida na época da semeadura e de uma pulverização de inseticida durante o seu desenvolvimento. 
O modelo desenvolvido avaliou as áreas a serem pulverizadas em cada mês para cada cultura. Para a seleção do conjunto mecanizado, o modelo de programação linear inteiro proposto considerou o ritmo operacional da propriedade. O ritmo operacional levou em consideração o período de tempo disponível para a realização da operação agrícola, sendo calculado de acordo com o número de domingos, feriados e dias úmidos (MIALHE, 1974).

O modelo de programação linear desenvolvido avaliou 20 modelos de pulverizadores disponíveis no mercado, sendo quatro autopropelidos, oito de arrasto e oito do tipo montado no engate de três pontos de tratores agrícolas (Tabela 1). Foi associado um código para cada modelo de pulverizador.

A largura nominal de trabalho, a capacidade do tanque, a velocidade de trabalho e o tipo de cada modelo foram obtidos nos prospectos dos fabricantes. A largura efetiva foi calculada a partir da largura nominal de trabalho do pulverizador e da largura da faixa de sobreposição da aplicação (MIALHE, 1974). Adotou-se uma faixa de sobreposição de 0,5 m.

Os preços foram obtidos de AGRIANUAL (2002) e consulta aos fabricantes dos pulverizadores, para o caso dos pulverizadores autopropelidos. A capacidade de campo operacional (MIALHE, 1974) foi calculada a partir dos dados dimensionais de cada pulverizador e das estimativas de eficiência de pulverização apresentadas por ASAE (1998) e HANNA (2002).

A eficiência de pulverização é dependente da estrutura fundiária e dos tempos perdidos nas manobras, no reabastecimento, na calibração e na limpeza dos bicos pulverizadores, dentre outros. É um fator de extrema importância que afeta diretamente a capacidade de campo operacional e, portanto, os custos; no entanto, é um tema que carece de maiores estudos para os diversos cenários agrícolas brasileiros e que não é o objeto do presente trabalho.

TABELA 1. Código, preço, capacidade de campo operacional, capacidade do tanque e tipo para cada modelo de pulverizador de barras utilizado.

\begin{tabular}{crrccc}
\hline Código & \multicolumn{1}{c}{$\begin{array}{c}\text { Preço } \\
(\mathrm{US} \$)^{1}\end{array}$} & $\begin{array}{c}\text { Largura Efetiva } \\
(\mathrm{m})\end{array}$ & $\begin{array}{c}\text { Capacidade de Campo } \\
\text { Operacional }\left(\mathrm{ha} \mathrm{h}^{-1}\right)\end{array}$ & $\begin{array}{c}\text { Capacidade do } \\
\text { Tanque }(\mathrm{L})\end{array}$ & $\begin{array}{c}\text { Tipo de } \\
\text { Pulverizador }\end{array}$ \\
\hline a1 & $100.000,00$ & 24,5 & 25,36 & 3.000 & autopropelido \\
a2 & $165.000,00$ & 26,5 & 36,57 & 3.028 & autopropelido \\
a3 & $100.000,00$ & 22,5 & 23,29 & 3.000 & autopropelido \\
a4 & $62.400,00$ & 21,0 & 19,85 & 2.000 & autopropelido \\
b1 & $5.082,00$ & 11,5 & 7,48 & 1.500 & arrasto \\
b2 & $1.620,00$ & 11,5 & 5,23 & 400 & montado \\
b3 & $1.662,00$ & 11,5 & 5,23 & 600 & montado \\
j1 & $1.924,00$ & 9,0 & 4,10 & 600 & montado \\
j2 & $1.374,00$ & 10,0 & 4,55 & 400 & montado \\
j3 & $2.585,00$ & 12,0 & 5,46 & 800 & montado \\
j4 & $4.140,00$ & 12,0 & 7,80 & 2.000 & arrasto \\
j5 & $10.452,00$ & 18,0 & 11,34 & 2.000 & arrasto \\
j6 & $2.192,00$ & 12,0 & 5,46 & 600 & montado \\
j7 & $4.499,00$ & 12,0 & 7,80 & 2.000 & arrasto \\
j8 & $2.488,00$ & 14,0 & 6,37 & 600 & montado \\
j9 & $11.643,00$ & 18,0 & 12,42 & 3.000 & arrasto \\
j10 & $16.717,00$ & 18,0 & 12,42 & 3.000 & arrasto \\
j11 & $15.198,00$ & 18,0 & 11,34 & 2.000 & arrasto \\
j12 & $22.968,00$ & 25,0 & 17,25 & 3.000 & arrasto \\
j13 & $1.628,00$ & 9,0 & 4,10 & 400 & montado \\
\hline
\end{tabular}

${ }^{1}$ Fonte: AGRIANUAL (2002). Taxa de câmbio utilizada R\$ 2,50 US\$ 
Para o desenvolvimento do modelo, foram realizados alguns cálculos preliminares à otimização, subdivididos em quatro etapas: cálculo do ritmo operacional; da capacidade de campo operacional; dos custos fixos e variáveis, e do número de conjuntos necessários para atender à necessidade do ritmo operacional. Considerou-se o custo fixo, tanto do pulverizador, quanto do trator agrícola. O custo fixo foi calculado segundo a metodologia descrita por BALASTREIRE (1990), sendo incluídas as taxas de abrigo, de seguro, de juros e de depreciação. A taxa de juros considerada foi de $8 \%$ ao ano, e a vida útil do equipamento foi de $2.300 \mathrm{~h}$ (ROTZ, 1986 e BALASTREIRE, 1990), trabalhando 300 horas ao ano, de acordo com o número de pulverizações necessárias para as culturas do milho e da soja. Segundo OLIVEIRA \& MILAN (2001), é mais apropriado avaliar a vida útil de uma máquina em horas e não em anos. Para a obtenção do custo operacional, foram incluídos as taxas de reparo e de manutenção (BALASTREIRE, 1990) e os custos com combustível e operador (AGRIANUAL, 2002). O consumo de combustível de uma máquina agrícola está diretamente relacionado com sua potência disponível. O consumo de combustível do trator foi estimado por meio de cálculos matemáticos sugeridos por BALASTREIRE (1990).

Os custos referentes ao trator agrícola foram considerados e somados aos custos referentes aos pulverizadores. Foram inclusos os custos referentes a um trator agrícola de $80 \mathrm{~kW}(108,8 \mathrm{cv}) \mathrm{de}$ potência, que é capaz de manter em operação qualquer um dos pulverizadores agrícolas selecionados. Considerou-se um valor de compra desse trator de US\$22.000,00 (AGRIANUAL, 2002). Porém, como foram considerados dois grupos de pulverizadores, os autopropelidos e os tratorizados, foi necessário criar-se um trator "virtual" com valor de compra igual a zero, pois os pulverizadores autopropelidos não necessitam de um trator agrícola para o seu funcionamento.

Uma representação esquemática da seqüência de cálculos para o ritmo operacional é ilustrada na Figura 1. Na Figura 2, ilustra-se a seqüência de cálculos para a obtenção do custo operacional.

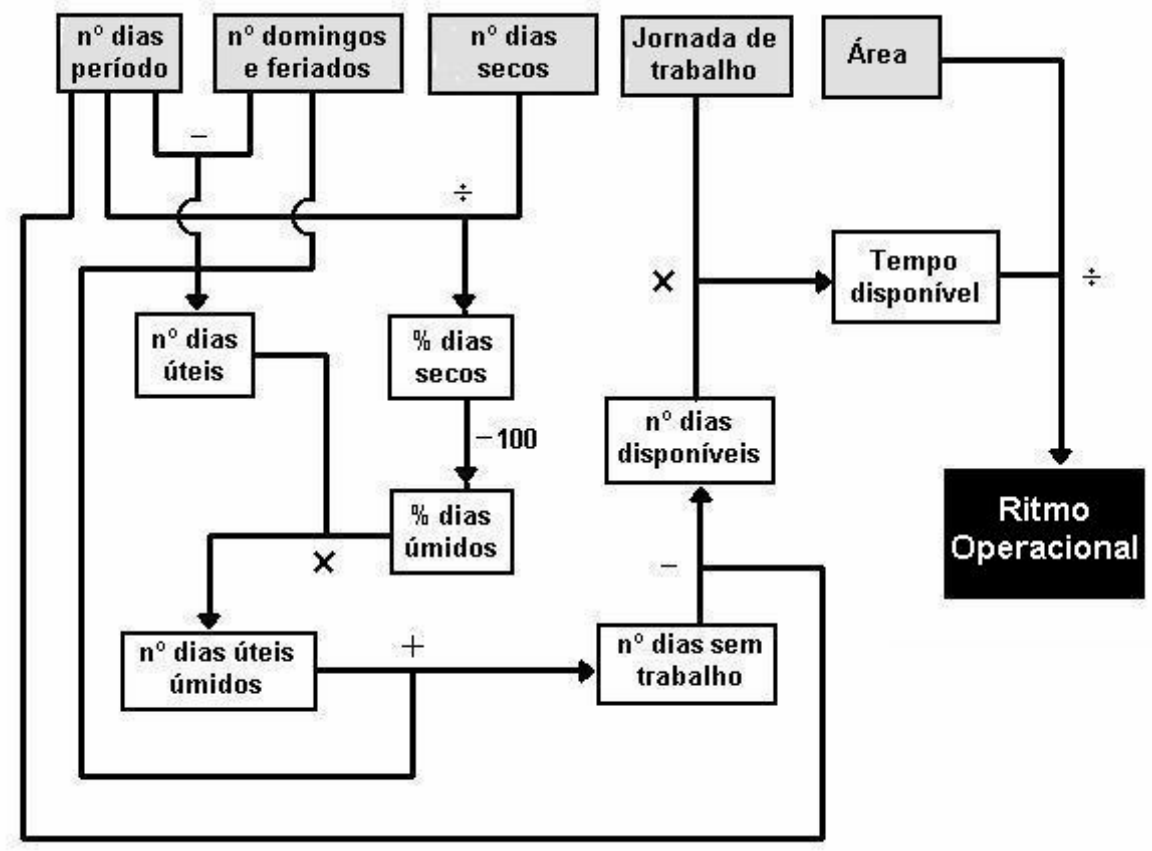

FIGURA 1. Representação esquemática da seqüência de cálculos para o ritmo operacional. 


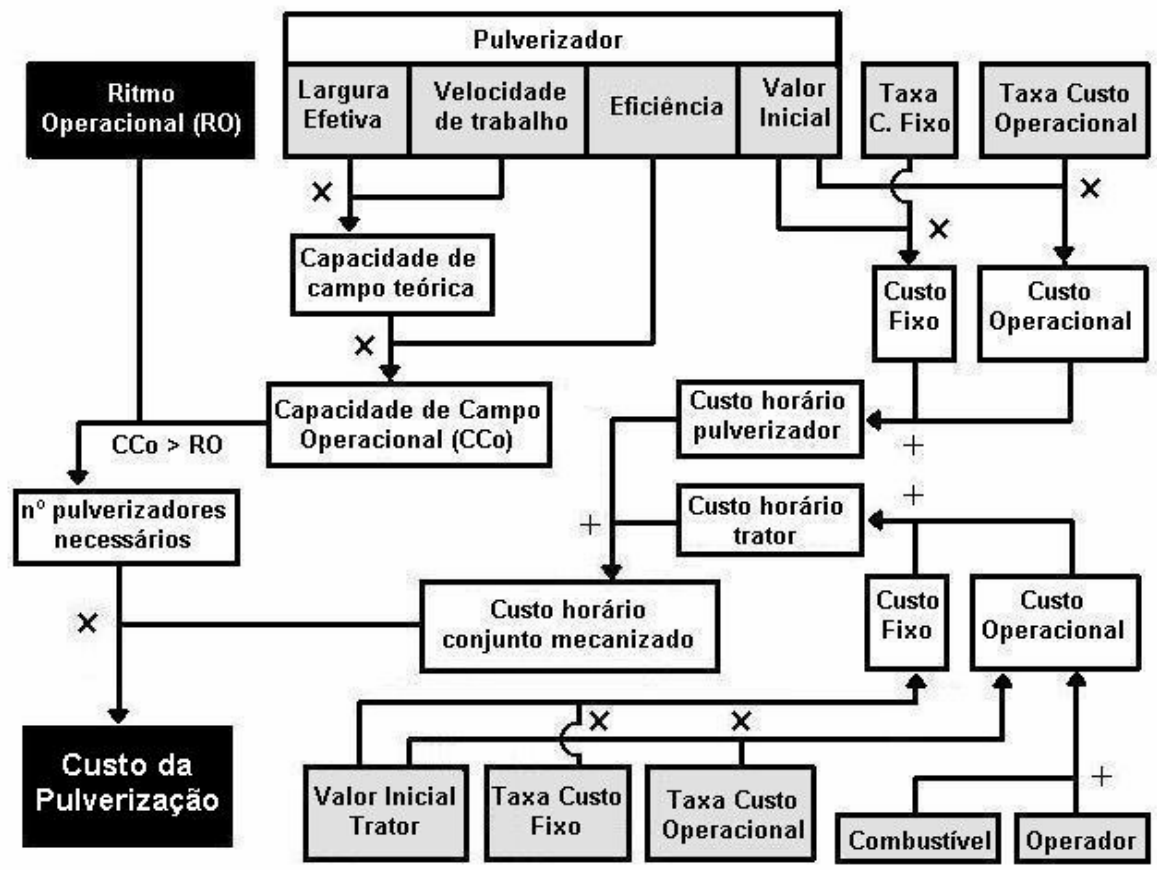

FIGURA 2. Representação esquemática da sequiência de cálculos para a definição do custo operacional.

O processamento do modelo de programação linear foi realizado por um computador K6-II de $450 \mathrm{MHz}$, com $128 \mathrm{Mb}$ de memória RAM. Foi utilizado o programa computacional Gams, versão estudantil 2.0.13, com a opção MIP (Mixed Integer Programming) do solver OSL2, o qual trabalha com programação linear inteira.

Utilizou-se o custo horário como fator de minimização no modelo, pois esse foi calculado baseado em todos os custos fixos e operacionais. No entanto, também foram calculados os custos por unidade de área (MIALHE, 1974) para fins comparativos.

Também foi realizada uma análise de sensibilidade para o custo horário com variação nos componentes dos custos operacionais, na área cultivada, no tempo disponível para o trabalho e na jornada de trabalho. De acordo com NORONHA (1981), ao fazer a análise de sensibilidade, procura-se modificar apenas uma variável de cada vez, deixando as demais nos níveis originais e pressupondo-se que cada variável afeta o resultado independentemente das demais.

\section{RESULTADOS E DISCUSSÃO}

O modelo matemático desenvolvido para o auxílio na tomada de decisão para a seleção de pulverizadores agrícolas de barras, com base no menor custo horário, foi:

$$
\operatorname{Min} \mathrm{C}=\sum_{\mathrm{k}=1}^{20} \sum_{\mathrm{l}=1}^{2} \mathrm{x}_{\mathrm{k}, \mathrm{l}}\left(\operatorname{pin}_{\mathrm{k}}\left(\mathrm{chi}_{\mathrm{k}}+\mathrm{cht}_{\mathrm{l}}\right)\right)
$$

em que,

$\mathrm{C}$ - custo horário, $\mathrm{US} \$ \mathrm{~h}^{-1}$;

$\mathrm{x}_{\mathrm{k}, \mathrm{l}}$ - conjunto mecanizado formado pelo pulverizador $\mathrm{k}$ e o trator 1 ;

$\operatorname{pin}_{\mathrm{k}}$ - número de pulverizadores necessários;

chi $_{\mathrm{k}}$ - custo horário total do pulverizador $\mathrm{k}, \mathrm{US} \$ \mathrm{~h}^{-1}$, e

$\mathrm{cht}_{1}$ - custo horário total do trator $1, \mathrm{US} \$ \mathrm{~h}^{-1}$. 
As restrições impostas ao modelo foram:

- número mínimo de conjuntos selecionados:

$\sum_{k=1}^{20} \sum_{\mathrm{l}=1}^{2} \mathrm{x}_{\mathrm{k}, \mathrm{l}} \geq 1$

- número máximo de operadores disponíveis na propriedade:

$$
\sum_{\mathrm{k}=1}^{20} \mathrm{x}_{\mathrm{k}, 1} \operatorname{pin}_{\mathrm{k}} \leq 4
$$

- trator "virtual" somente pode ser selecionado quando um pulverizador autopropelido for selecionado:

$$
\begin{aligned}
& \mathrm{x}_{\mathrm{b} 1 \text {, virtual }}+\mathrm{x}_{\mathrm{b} 2 \text {, virtual }}+\mathrm{x}_{\mathrm{b} 3 \text {, virtual }}+\mathrm{x}_{\mathrm{j} 1 \text {, virtual }}+\mathrm{x}_{\mathrm{j} 2 \text {, virtual }}+\mathrm{x}_{\mathrm{j} 3 \text {, virtual }}+ \\
& \mathrm{X}_{\mathrm{j} 4 \text {, virtual }}+\mathrm{X}_{\mathrm{j} 5 \text {, virtual }}+\mathrm{X}_{\mathrm{j} 6 \text {, virtual }}+\mathrm{X}_{\mathrm{j} 7 \text {, virtual }}+\mathrm{X}_{\mathrm{j} 8 \text {, virtual }}+\mathrm{X}_{\mathrm{j} \text { 9, virtual }}+ \\
& \mathrm{X}_{\mathrm{j} 10 \text {,virtual }}+\mathrm{X}_{\mathrm{j} 11 \text {,virtual }}+\mathrm{X}_{\mathrm{j} 12 \text {, virtual }}+\mathrm{X}_{\mathrm{j} 13 \text {, virtual }}=0 \\
& \text { - restrição binária: } \\
& \mathrm{X}_{\mathrm{k}, 1}=0 \text { ou } 1 \text {, para } \mathrm{k}=1,2, \ldots, 20 \text { e } \mathrm{l}=1,2
\end{aligned}
$$

A solução gerada pelo programa foi considerada ótima, significando que a função-objetivo pôde ser minimizada. O sistema resolveu o problema em 0,01 s.

O relaxamento da restrição do número máximo de operadores não mudou a solução ótima para o problema. A restrição do número máximo de operadores apresentou folga, não sendo uma restrição atuante.

O valor da função-objetivo encontrado foi de US\$16,05 $\mathrm{h}^{-1}$. O custo por unidade de área para o conjunto selecionado foi de US\$ $0,93 \mathrm{ha}^{-1}$. Para atingir esse valor, foi selecionado somente um conjunto mecanizado pulverizador+trator. O pulverizador selecionado, código j12, possui largura efetiva de $25 \mathrm{~m}$, capacidade do tanque de 3.000 L e é um pulverizador de arrasto.

Verificou-se que, ao reduzir o tempo disponível para a pulverização no mês para duas semanas, o modelo selecionou outro pulverizador devido ao menor custo horário. Esse fato também ocorreu ao reduzir o tempo disponível para uma semana. Na Tabela 2, apresenta-se a análise de sensibilidade para o custo horário devido à variação do tempo disponível para as pulverizações nas culturas da soja e do

\begin{tabular}{|c|c|c|c|c|c|}
\hline $\begin{array}{c}\text { Tempo } \\
\text { Disponível }\end{array}$ & $\begin{array}{l}\text { Ritmo Operacional } \\
\left(\mathrm{ha} \mathrm{h}^{-1}\right)\end{array}$ & $\begin{array}{l}\text { Código do Conjunto } \\
\text { Selecionado }\end{array}$ & $\begin{array}{l}\text { Número de } \\
\text { Conjuntos }\end{array}$ & $\begin{array}{l}\text { Custo Horário } \\
\left.\text { (US\$ h }{ }^{-1}\right)\end{array}$ & $\begin{array}{l}\text { Custo por Área } \\
\left(\text { US } \$ \mathrm{ha}^{-1}\right)\end{array}$ \\
\hline 4 semanas & 13,55 & j12 & 1 & 16,05 & 0,93 \\
\hline 2 semanas & 27,10 & j12 & 2 & 32,10 & 1,86 \\
\hline 1 semana & 54,20 & $\mathrm{a} 4^{1}$ & 3 & 62,63 & 3,15 \\
\hline
\end{tabular}
milho, na propriedade hipotética. Pode-se observar que o custo horário dobrou quando o tempo disponível foi reduzido pela metade, pois o modelo dobrou o número de conjuntos mecanizados para atender ao ritmo operacional máximo no período.

TABELA 2. Análise de sensibilidade para o custo horário devido à variação do tempo disponível para a operação agrícola de pulverização.

${ }^{1}$ Pulverizador autopropelido. 
A alteração da jornada de trabalho também afetou o conjunto mecanizado selecionado (Tabela 3), porém o número de conjuntos selecionados permaneceu inalterado. Verifica-se que o custo horário diminuiu quando houve um acréscimo na jornada de trabalho, pois a diminuição do ritmo operacional permitiu a seleção de um pulverizador com menor capacidade de campo operacional e, portanto, mais barato. Entretanto, um conjunto mecanizado com menor capacidade de campo operacional necessita trabalhar um número de horas maior para pulverizar a mesma área, fazendo com que o custo por unidade de área se elevasse, como é observado na Tabela 3. Apesar disso, é menor o tempo ocioso desse pulverizador na propriedade, como também é menor o seu custo de aquisição.

TABELA 3. Análise de sensibilidade para o custo horário devido à variação da jornada de trabalho para a operação agrícola de pulverização.

\begin{tabular}{cccccc}
\hline $\begin{array}{c}\text { Jornada } \\
\text { (horas) }\end{array}$ & $\begin{array}{c}\text { Ritmo Operacional } \\
\left(\mathrm{ha} \mathrm{h}^{-1}\right)\end{array}$ & $\begin{array}{c}\text { Código do Conjunto } \\
\text { Selecionado }\end{array}$ & $\begin{array}{c}\text { Número de } \\
\text { Conjuntos }\end{array}$ & $\begin{array}{c}\text { Custo Horário } \\
\left.\left(\mathrm{US}^{-1}\right)^{-1}\right)\end{array}$ & $\begin{array}{c}\text { Custo por Área } \\
\left(\mathrm{US} \mathrm{ha}^{-1}\right)\end{array}$ \\
\hline 8 & 13,55 & $\mathrm{j} 12$ & 1 & 16,05 & 0,93 \\
10 & 10,84 & $\mathrm{j} 5$ & 1 & 12,58 & 1,11 \\
12 & 9,03 & $\mathrm{j} 5$ & 1 & 12,58 & 1,11 \\
14 & 7,74 & $\mathrm{j} 4$ & 1 & 10,83 & 1,39 \\
16 & 6,77 & $\mathrm{j} 4$ & 1 & 10,38 & 1,39 \\
\hline
\end{tabular}

Foi verificado que, para as condições impostas, um pulverizador autopropelido apenas foi escolhido quando foi alterada a área para 4.000 ha, devido a sua maior capacidade operacional (Tabela 4). Também se verificou que, quando a área foi superior a 6.000 ha, um relaxamento na restrição do número máximo de operadores na propriedade proporcionou a seleção de conjuntos mecanizados com um menor custo horário. Se o modelo de programação linear desenvolvido fosse baseado na minimização do custo por unidade de área (US\$ ha ${ }^{-1}$ ), esse selecionaria o conjunto mecanizado pulverizador + trator j12, se a área fosse menor que 500 ha. Esse conjunto corresponde ao pulverizador de arrasto com largura efetiva de $25 \mathrm{~m}$, com capacidade do tanque de $3.000 \mathrm{~L}$ e com custo por unidade de área de 0,93 US\$ ha ${ }^{-1}$. Todavia, esse é um equipamento incompatível para uma propriedade com área de 50 ou 10 ha, por exemplo, pois possui uma capacidade de campo operacional muito maior que aquela exigida pelo ritmo operacional (Tabela 4).

TABELA 4. Análise de sensibilidade para o custo horário devido à variação da área a ser pulverizada.

\begin{tabular}{|c|c|c|c|c|c|}
\hline $\begin{array}{l}\text { Área } \\
\text { (ha) }\end{array}$ & $\begin{array}{c}\text { Ritmo Operacional } \\
\left(\text { ha h }^{-1}\right)\end{array}$ & $\begin{array}{c}\text { Código do Conjunto } \\
\text { Selecionado }\end{array}$ & $\begin{array}{l}\text { Número de } \\
\text { Conjuntos }\end{array}$ & $\begin{array}{c}\text { Custo Horário } \\
\left(\mathrm{US} \$ \mathrm{~h}^{-1}\right)\end{array}$ & $\begin{array}{l}\text { Custo por Área } \\
\left(\mathrm{US} \$ \mathrm{ha}^{-1}\right)\end{array}$ \\
\hline 10 & 0,14 & $\mathrm{j} 2$ & 1 & 10,07 & 2,21 \\
\hline 50 & 0,68 & $\mathrm{j} 2$ & 1 & 10,07 & 2,21 \\
\hline 100 & 1,36 & $\mathrm{j} 2$ & 1 & 10,07 & 2,21 \\
\hline 500 & 6,77 & j4 & 1 & 10,83 & 1,39 \\
\hline 800 & 10,84 & $\mathrm{j} 5$ & 1 & 12,58 & 1,11 \\
\hline 1.000 & 13,55 & $\mathrm{j} 12$ & 1 & 16,05 & 0,93 \\
\hline 2.000 & 27,10 & j12 & 2 & 32,10 & 0,93 \\
\hline 3.000 & 40,65 & j12 & 3 & 48,15 & 0,93 \\
\hline 4.000 & 54,20 & $\mathrm{a} 4^{1}$ & 3 & 62,63 & 1,05 \\
\hline 5.000 & 67,75 & j12 & 4 & 64,21 & 0,93 \\
\hline 6.000 & 81,29 & $a 3^{1}$ & 4 & 108,95 & 1,17 \\
\hline 6.000 & 81,29 & $\mathrm{j} 12$ & 5 & 80,26 & 0,93 \\
\hline
\end{tabular}

${ }^{1}$ Pulverizador autopropelido. 
A análise de sensibilidade para o custo horário, com a variação em $10 \%$ nos valores dos custos operacionais, mostrou que a taxa de reparo do maquinário agrícola é o fator que mais afetou o custo horário (Figura 3), seguido da taxa de depreciação, concordando com BARBOZA et al. (1997) e OLIVEIRA \& MILAN (2001). Isso mostra a importância da renovação da frota agrícola para não promover o seu sucateamento e aumentar os custos operacionais. Assim, os custos de reparo e de depreciação são importantes indicadores do momento ótimo para a substituição do conjunto mecanizado. Segundo OLIVEIRA \& MILAN (2001), qualquer máquina poderia ter a sua vida útil prolongada, se não fosse o fato do obsoletismo e dos altos custos operacionais que tornam antieconômico o uso dessa máquina a partir de um determinado instante. Verificou-se que, ao elevar o preço inicial do conjunto mecanizado pulverizador + trator em $10 \%$, houve elevação no custo horário em $6,9 \%$, sendo o fator de entrada que mais interferiu na variação do custo horário final. Foi observado que a variação em $10 \%$ nos valores dos outros componentes dos custos (área pulverizada, tempo disponível e jornada de trabalho) não alterou o custo horário, pois o conjunto selecionado permaneceu inalterado.

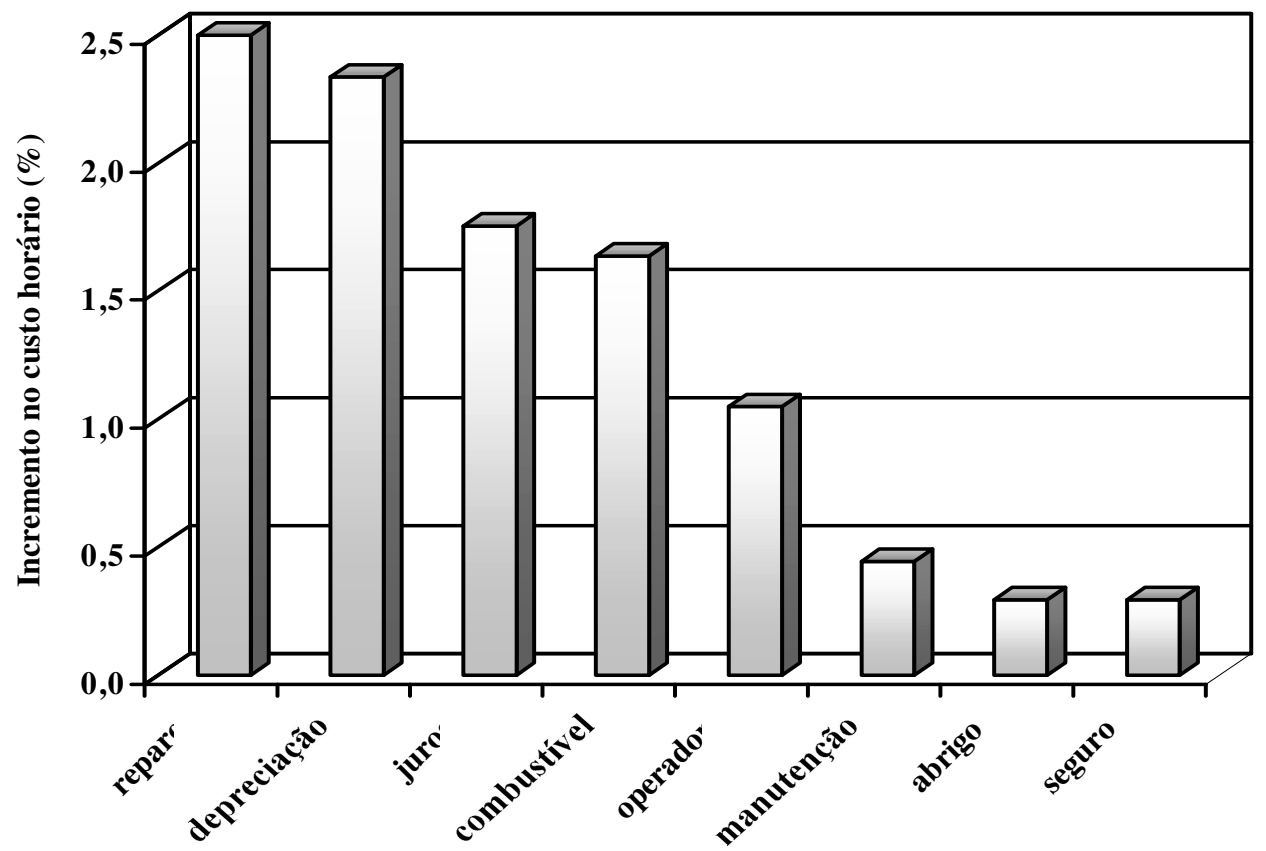

FIGURA 3. Análise de sensibilidade para o custo horário (\%) devido à variação em $10 \%$ nos valores dos custos operacionais.

\section{CONCLUSÕES}

O modelo de programação linear binário desenvolvido permitiu a otimização da função-objetivo, auxiliando na seleção de pulverizadores agrícolas com base no menor custo horário.

Para a propriedade hipotética em estudo, o valor da função-objetivo encontrado foi de US\$ $16,05 \mathrm{~h}^{-1}$. O custo por unidade de área para o conjunto selecionado foi de US\$ $0,93 \mathrm{ha}^{-1}$.

A análise de sensibilidade dos componentes dos custos operacionais mostrou que as taxas de reparo e depreciação foram os fatores que mais interferiram na variação do custo horário dos conjuntos avaliados. O preço de aquisição do conjunto mecanizado foi o fator de entrada que mais interferiu na variação do custo horário final.

O modelo matemático desenvolvido facilitou a realização da análise de sensibilidade, que foi processada em um tempo muito pequeno. 


\section{REFERÊNCIAS BIBLIOGRÁFICAS}

AGRIANUAL 2002. São Paulo: FNP Consultoria \& Comércio, 2003. p.84-93.

ASAE. Agricultural machinery management data. St. Joseph: ASAE Standards, 1998. p.360-6.

BALASTREIRE, L.A. Máquinas agrícolas. São Paulo: Manole, 1990. 307 p.

BARBOZA, M.M.; MILAN, M.; COELHO, J.L.D. Planning of an agricultural machinery system. In: WORLD CONGRESS ON COMPUTERS IN AGRICULTURE AND NATURAL RESOURCES, 2 ., 1997, St. Joseph. Proceedings... St. Joseph: ASAE, 1997. p.137-41.

CAIXETA FILHO, J.V. Pesquisa operacional. São Paulo: Atlas, 2001. 171 p.

FEY, E.; CATARINA, A.S.; SANTOS, S.R.; PEREIRA, J.O. Planejamento de um sistema agrícola utilizando programação linear: estudo de caso. Engenharia Agrícola, Jaboticabal, v.22, n.3, p.368-77, 2002.

HANNA, M. Estimating the field capacity of farm machines. Net, Iowa. Disponível em: $<$ http://www.extension.iastate.edu/agdm/crops/pdf/a3-24.pdf>. Acesso em: 17 abril 2002.

HILLER, F.; LIEBERMAN, G.J. Introdução à pesquisa operacional. 3.ed. São Paulo: Edusp, 1988. $803 \mathrm{p}$.

LOPES, J.D.S.; MANTOVANI, E.C.; PINTO, F.A.C.; QUEIROZ, D.M. Desenvolvimento de um programa computacional para selecionar, economicamente, um sistema de mecanização agrícola. Pesquisa Agropecuária Brasileira, Brasília, v.30, n.4, p.537-42. 1995.

MERCANTE, E.; GABRIEL, A.; SOUZA FILHO, E.G.; OPAZO, M.A.U.; WRONSKI, F.; ANTES, C.A.; SORDI, C.; MAGGI, M.F.; JOHANN, L.A. Software para seleção e estimativa de custo operacional de máquinas e implementos agrícolas. In: CONGRESSO BRASILEIRO DE ENGENHARIA AGRÍCOLA, 30., 2001, Foz do Iguaçu. Anais... Cascavel: Sociedade Brasileira de Engenharia Agrícola, 2001. 1 CD ROM.

MIALHE, L.G. Manual de mecanização agrícola. São Paulo: Ceres, 1974. 301 p.

NORONHA, J.F. Projetos agropecuários: administração financeira, orçamento e viabilidade econômica. São Paulo: Atlas, 1981. 274 p.

OLIVEIRA, M.D.M.; MILAN, M. Custo operacional e ponto de renovação de tratores agrícolas de pneus: avaliação de uma frota. In: CONGRESSO BRASILEIRO DE ENGENHARIA AGRÍCOLA, 30., 2001, Foz do Iguaçu. Anais... Cascavel: Sociedade Brasileira de Engenharia Agrícola, 2001. 1 CD ROM

ROTZ, C.A. A standard model for repair costs of agricultural machinery. St. Joseph: ASAE, 1986. 7p. (Paper no 86-1527)

SOFFNER, R.K.; MILAN, M.; RÍPOLI, T.C.C. Gerenciamento global de sistema agrícola em unidades sucroalcooleiras através de programação linear. Stab - Açúcar, Álcool e Derivados, Piracicaba, v.11, n.5, p.16-20, 1993.

WILLIAMS, H.P. Model building in mathematical programming. 3.ed. Chichester: Wiley \& Sons, 1993. $353 \mathrm{p}$. 